\section{SERVIÇOS DE ATENÇÃO DOMICILIAR: CRITÉRIOS DE ELEGIBILIDADE, INCLUSÃO, EXCLUSÃO E ALTA}

\author{
Home care services: eligibility, inclusion, exclusion and \\ discharge criteria
}

\author{
Servicios de atención domiciliaria: criterios de elegibilidad, \\ inclusión, exclusión y alta
}

\section{RESUMO}

Objetivo: Descrever os aspectos que envolvem a elegibilidade, inclusão, exclusão e alta na atenção domiciliar na perspectiva de gestores e coordenadores de atenção domiciliar. Métodos: Trata-se de estudo descritivo-exploratório de abordagem qualitativa. Foram realizadas entrevistas com cinco gestores e 17 coordenadores de 16 municípios de Minas Gerais entre janeiro e setembro de 2015. Resultados: Os entrevistados afirmaram seguir critérios propostos nas diretrizes nacionais do serviço de atenção domiciliar, com algumas exceções. No que diz respeito à elegibilidade, a maioria dos gestores informa que os pacientes passam por avaliação clínica realizada pelo médico que define se o paciente segue para a atenção domiciliar ou não. Os critérios de inclusão referem-se à avaliação das condições do paciente, a identificação do cuidador, a questão socioambiental, o acesso da equipe e adaptação da residência às necessidades do paciente. Como critérios de exclusão, apontam as condições estruturais do domicílio, a ausência de cuidador e de segurança para os profissionais. A alta é referenciada por critérios de estabilidade clínica e o fim do tratamento. Conclusão: Embora os critérios dos serviços de assistência domiciliar estejam definidos pelo Ministério da Saúde, os gestores e coordenadores abrem exceções na inclusão e alta considerando: a fragilidade da rede para alta e encaminhamento para outro ponto de atenção e a realidade do paciente. A atenção domiciliar deve ser realizada quando as condições clínicas e administrativas do usuário permitam, considerando os critérios de elegibilidade, inclusão, exclusão e alta.

Descritores: Assistência Domiciliar; Serviços de Assistência Domiciliar; Regionalização.

\section{ABSTRACT}

Objective: To describe the aspects involving eligibility, inclusion, exclusion and discharge in home care from the perspective of home care managers and coordinators. Methods: This is qualitative descriptive exploratory study. Interviews were conducted with five managers and 17 coordinators from 16 municipalities of Minas Gerais between January and September 2015. Results: Respondents reported following the criteria proposed in the national guidelines of the home care service, but with some exceptions. With regard to eligibility, most managers reported that patients undergo clinical evaluation performed by the physician who determines whether or not the patient is eligible for home care. Inclusion criteria refer to the assessment of the patient's conditions, the identification of the caregiver, social and environmental issues, team access and adaptation of the home to the patient's needs. Exclusion criteria point to the structural conditions of the home, the lack of caregiver and safety for professionals. The discharge is referenced by clinical stability criteria and the end of treatment. Conclusion: Although home care services criteria are set by the Ministry of Health, managers and coordinators make exceptions to the inclusion and discharge criteria considering: the fragility of the network regarding discharge and referral to another point of care and the patient's reality. Home care should be performed when the clinical and administrative conditions of the user are just right, considering the eligibility, inclusion, exclusion and discharge criteria.

Descriptors: Home Care; Home Care Services; Regional Health Planning.
Artigo Original
1) Universidade Estadual de Montes Claros - Unimontes - Montes Claros (MG) - Brasil

2) Universidade Federal de Minas Gerais UFMG - Belo Horizonte (MG) - Brasil
Recebido em: 22/04/2016 Revisado em: $19 / 05 / 2016$ Aceito em: 26/06/2016 


\section{RESUMEN}

Objetivo: Describir los aspectos que involucran la elegibilidad, la inclusión, la exclusión y la alta de la atención domiciliaria desde la perspectiva de los gestores y coordinadores de la atención domiciliaria. Métodos: Se trata de un estudio descriptivo-exploratorio de abordaje cualitativo. Fueron realizadas entrevistas con cinco gestores y 17 coordinadores de 16 municipios de Minas Gerais entre enero y septiembre de 2015. Resultados: Los entrevistados afirmaron que con algunas excepciones seguian los criterios propuestos en las directrices nacionales del servicio de atención domiciliaria. Respecto a la elegibilidad, la mayoría de los gestores relata que los pacientes pasan por una evaluación clínica realizada por el médico el cual define si el paciente sigue para la atención domiciliaria $o$ no. Los criterios de inclusión se refieren a la evaluación de las condiciones del paciente, la identificación del cuidador, la cuestión socioambiental, el acceso del equipo y la adaptación de la vivienda a las necesidades del paciente. Los criterios de exclusión fueron las condiciones estructurales del domicilio, la ausencia del cuidador y de seguridad para los profesionales. La alta es referenciada por criterios de estabilidad clínica y el fin del tratamiento. Conclusión: Aunque los criterios de los servicios de atención domiciliaria son definidos por el Ministerio de la Salud, los gestores y coordinadores permiten excepciones para la inclusión y la alta por considerar: la fragilidad de la red para la alta y el encaminamiento para otro sitio de atención y la realidad del paciente. La atención domiciliaria debe ser realizada cuando las condiciones clínicas y administrativas del usuario permitan, considerando los criterios de elegibilidad, inclusión, exclusión y alta.

Descriptores: Atención Domiciliaria de Salud; Servicios de Atención de Salud a Domicilio; Regionalización.

\section{INTRODUÇÃO}

O Brasil passa por diversas transformações e entre estas se destaca a transição epidemiológica e demográfica. Estas modificações, atreladas ao aumento do envelhecimento populacional, com a rápida queda da fecundidade e redução da mortalidade, provocam mudanças nos padrões de morte, morbidade e invalidez associados aos fatores biológicos, econômicos, ambientais, científicos e culturais ${ }^{(1,2)}$.

Decorrente dessa transição, até a década de 30 do século XXI haverá predomínio de idosos em relação ao número de crianças, além de considerável diminuição da população em idade produtiva ${ }^{(3)}$. Esse aumento faz com que haja maior procura por serviços de saúde, hospitalizações mais frequentes e maior tempo de ocupação do leito considerando as características da faixa etária. Para conseguir lidar com estas mudanças, são necessárias novas estratégias e mecanismos de cuidado em saúde, além da construção de políticas públicas adequadas de atenção à saúde e intervenções integrais que se desvinculem apenas do cuidado hospitalar e de práticas anti-hegemônicas ${ }^{(4)}$.

A assistência domiciliar, desta forma, assume lugar como uma das alternativas de cuidado, com motivação inicial de descongestionar os hospitais e proporcionar a construção de nova lógica de atenção, com enfoque em ambiente psicossocial mais favorável aos pacientes e familiares com promoção e prevenção à saúde e na humanização da atenção ${ }^{(5)}$. A organização de serviços com foco no domicílio não é uma prática recente, surgiu nos Estados Unidos em 1947, no Brasil em 1949 e vem se expandindo mundialmente ${ }^{(6)}$.

Devido à relevância dessa modalidade de cuidado, o Ministério da Saúde institui, no âmbito do Sistema Único de Saúde (SUS), a Internação Domiciliar, como um conjunto de atividades prestadas no domicílio, por meio da Portaria MS n ${ }^{\circ} 2.529$ de 19 de outubro de 2006 ${ }^{(7)}$. Em 2011, instituiu o Programa de Atenção Domiciliar (PAD), também conhecido como Melhor em Casa, por meio da Portaria MS $n^{\circ} 2.029$, que apresentava normas para habilitação e cadastro no Serviço de Atenção Domiciliar (SAD) ${ }^{(8)}$. Este programa sofreu modificações e atualmente é regido pela Portaria MS $n^{\circ} 963$ de 27 de maio de $2013^{(9)}$.

O programa se apresenta estruturado com equipe multidisciplinar com o foco de atender o paciente na sua integralidade com o maior e melhor nível de resposta. Esta é dividida em equipe multiprofissional de atenção domiciliar (EMAD) e equipe multiprofissional de apoio (EMAP) ${ }^{(10)}$. Quanto à organização do atendimento domiciliar do usuário, três modalidades de atenção domiciliar (AD1, AD2 e AD3) ficam estabelecidas, conforme grau de complexidade e frequência das visitas domiciliares necessárias ${ }^{(10)}$.

$\mathrm{Na}$ atenção domiciliar, a promoção da saúde configurase estratégia propícia ao enfrentamento das múltiplas dificuldades de saúde que afetam as populações. Envolver os trabalhadores da saúde, gestores e usuários na busca por melhores condições de saúde, bem como conhecer os determinantes do contexto sobre o qual se pretende intervir é de fundamental importância ${ }^{(11)}$. Além de envolver a prática de políticas econômicas, sociais e de saúde, a atenção domiciliar objetiva reduzir os riscos de os indivíduos adoecerem; promove a fiscalização e o planejamento dos programas de saúde; e a execução das atividades preventivas. Abrange desde a promoção da saúde até a recuperação dos indivíduos acometidos por um agravo e que estão situados em seus respectivos domicílios ${ }^{(12)}$.

Para inserção e permanência do usuário no programa de $\mathrm{AD}$, são estabelecidos alguns critérios, a saber: critérios de elegibilidade, critérios de inclusão, critérios de exclusão e critérios de alta. Todos os critérios são baseados e aplicados 
considerando a Portaria MS n ${ }^{\circ} 963 / 2013$, que define o perfil do paciente a ser atendido ${ }^{(9)}$. Diante do exposto, perguntamse como esses critérios são incorporados nas equipes de atenção domiciliar?

Nesse sentido, a proposta deste artigo é analisar os aspectos que envolvem a elegibilidade, inclusão, exclusão e alta na atenção domiciliar na perspectiva de gestores em saúde e coordenadores de atenção domiciliar.

\section{MÉTODOS}

Trata-se de estudo de natureza qualitativa, com abordagem descritiva exploratória. O percurso metodológico do estudo teve como cenário os Serviços de Atenção Domiciliar (SAD) Minas Gerais (MG) que possuíam 20.000 ou mais habitantes em conformidade com a Portaria MS n 963 de 2013. Para identificação destes serviços, foi realizado o levantamento no Cadastro Nacional de Estabelecimentos de Saúde (CNES) no ano de 2015. Foi identificado que o Estado de MG é composto por $25 \mathrm{SAD}$. As entrevistas foram realizadas em 16 municípios.

Foram realizadas 22 entrevistas com cinco gestores em saúde dos municípios e 17 coordenadores, profissionais que assumiam o cargo de coordenação do SAD. Cada participante recebeu um código constituído por letra $\mathrm{M} \mathrm{e}$ número do município e $\mathrm{G}$ para os gestores e coordenadores, com a respectiva identificação numérica.

As entrevistas ocorreram no local de trabalho destes profissionais e foram orientadas por roteiro semiestruturado, com questões sobre o cargo, função e tempo de atuação do gestor ou coordenador. Para o conhecimento dos serviços de atenção domiciliar, foram indagados quais os critérios de elegibilidade, inclusão, exclusão e alta que os municípios adotam para Atenção Domiciliar. As entrevistas foram gravadas e, em seguida, transcritas para facilitar a análise das informações.

De forma complementar foi realizada análise do caderno do Melhor em Casa, volume 1, que discute sobre os critérios do PAD. Os cadernos do Melhor em Casa são manuais com orientações sobre a organização, o funcionamento, a estrutura e a composição do programa.

De posse do material, foi realizada a análise de conteúdo $^{(13)}$. Procedeu-se a leitura transversal do material empírico destacando-se as unidades de registro e as unidades de significação. Os dados coletados foram analisados em categorias agrupadas analogicamente. Neste caminho, com a utilização da análise de conteúdo surgiram duas categorias, sendo a primeira de Critérios de Elegibilidade e Inclusão e a segunda de Critérios de Exclusão e Alta.

Este estudo foi aprovado pelo Comitê de Ética em Pesquisa da Universidade Federal de Minas Gerais sob o parecer $\mathrm{n}^{\circ} 129.725$ por atender a todos os requisitos éticos necessários à pesquisa científica realizada com seres humanos, tais como a participação voluntária, a privacidade dos participantes e a confidencialidade das informações.

\section{RESULTADOS E DISCUSSÃO}

Foram realizadas entrevistas com cinco gestores e 17 coordenadores de 16 municípios de Minas Gerais entre janeiro e setembro de 2015. Dos 17 coordenadores do SAD entrevistados, 12 encontram-se no cargo de coordenador desde a criação do programa Melhor em Casa. Quatro coordenadores são do sexo masculino e 13 do sexo feminino e possuem as seguintes formações: 10 enfermeiros, três assistentes sociais e dois fisioterapeutas. Quanto aos gestores, três são do sexo feminino e dois do sexo masculino e possuem as seguintes formações, medicina, odontologia, enfermagem e ciências contábeis.

\section{Critérios de Elegibilidade e Inclusão}

Procedeu-se a leitura transversal do material empírico destacando-se as unidades de registro e as unidades de significação. Os dados coletados foram analisados em categorias agrupadas analogicamente. Neste caminho, com a utilização da análise de conteúdo surgiram duas categorias, sendo a primeira de Critérios de Elegibilidade e Inclusão e a segunda de Critérios de Exclusão e Alta.

De acordo com os documentos normativos do Programa Melhor em Casa, "os critérios e condições de elegibilidade são o conjunto de elementos e informações que permitem avaliar a inclusão do paciente na atenção domiciliar" ${ }^{\prime(8)}$. Os critérios de elegibilidade abrangem aspectos clínicos e administrativos. Os aspectos clínicos dizem respeito à situação do quadro do paciente, aos procedimentos assistenciais e a frequência de visitas. Os aspectos administrativos referem-se às condições operacionais e legais para que o atendimento do paciente seja realizado, o que inclui residir no município e possuir ambiente domiciliar minimamente adequado e seguro para receber o paciente. A referência do paciente pode advir de concordância e encaminhamento do médico assistente, seja na Atenção Básica, Serviço de Urgência e Emergência ou Hospital. Esta referência necessita de protocolo ou instrumento de contrarreferência, com relatório minucioso, contendo dados relevantes para avaliação do quadro clínico do usuário. Deve também possuir um cuidador quando o usuário se encontra com dependência funcional ${ }^{(8)}$.

Diante do exposto pelo programa, verifica-se que os relatos dos participantes referem os mesmos aspectos marcados na política no que se diz respeito aos aspectos clínicos e administrativos da determinação de elegibilidade do programa: 
"Nossos critérios de elegibilidade, nós solicitamos sempre um relatório médico com a história clínica do paciente... $e$ indicando o porquê dos cuidados domiciliares e do acompanhamento multiprofissional e nesse relatório nós solicitamos o CID primário e secundário, que é até uma solicitação do Ministério da Saúde que a gente lança depois no RAS” (M14P2)

"[...] socioambiental do paciente tem que trazer condições pra esses, pra esses pacientes ir pra casa. Então ele precisa ser uma área que não traga riscos pra equipe... uma área que não tenha tráfico de drogas, ninguém violento dentro da casa, que a gente consiga fazer a assistência domiciliar... uma área que não coloque em risco a saúde do paciente. Então, uma casa que esteja desabando, que não tenha condições dele morar, nenhum conteúdo de higiene dentro daquela casa” (M16G1)

"Então residir aqui, ou se manter aqui em algum lugar até que seja feita, ao longo do tratamento. Caso não tem autonomia de cuidado ele não tem a condição de aprender, esse paciente tem que ter um cuidador responsável e que esteja disposto a aprender, a se capacitar" (M15G1)

O determinante para elegibilidade do paciente, quanto aos critérios clínicos, refere-se ao quadro do paciente, sendo classificados em AD1, AD2 e AD3. A modalidade AD1 contempla pacientes com problemas de saúde controlados/ compensados, o que permite maior espaçamento entre as visitas, não necessitam de procedimentos e técnicas de maior complexidade e não necessitam de atendimentos médicos frequentes. Assim, os cuidados domiciliares a estes pacientes é responsabilidade das equipes de Estratégia Saúde da Família. Para que o paciente seja incluído no $\mathrm{SAD}$, ele precisa se encaixar no nível AD2 ou AD3, o que inclui a impossibilidade de deslocar para uma unidade de saúde e precisar de atendimento pelo menos uma vez por semana da equipe de saúde ${ }^{(8)}$.

Considerando o perfil clínico do paciente, a Portaria MS n 963 de 2013 destaca a inclusão de demandas por procedimentos de maior complexidade e que possam ser realizados no domicílio, tais como: curativos complexos e drenagem de abscesso, necessidade frequente de exames de laboratório de menor complexidade e dependência de monitoramento frequente de sinais vitais. Além do acompanhamento ponderal de baixo peso, da atenção nutricional e da medicação, são incluídos também os pacientes que necessitam de equipamentos/procedimentos de oxigenoterapia e suporte ventilatório não invasivo, Pressão Positiva Contínua nas Vias Aéreas (CPAP), Pressão Aérea Positiva por dois Níveis (BIPAP), concentrador de $\mathrm{O}_{2}$, diálise peritoneal e paracentese ${ }^{(10)}$. Para tal, deve ocorrer o treinamento do cuidador para que haja a adaptação do manuseio de dispositivos, sondas e ostomias, aspirador, pósoperatório, reabilitação. Este atendimento se faz oportuno aos pacientes até apresentarem condições de frequentarem serviços de reabilitação.

Segundo os coordenadores participantes, a avaliação e a classificação dos usuários são realizadas conforme preconizado pelo manual do PAD:

“... já vem direcionando ... nesse sentido aí faz essa avaliação nessa escala de baixa, média e alta complexidade, e nela a gente não cadastra os pacientes de baixa complexidade, que seriam na modalidade de AD1, que é perfil de unidade; e já a média e alta complexidade nessa avaliação” (M7G2)

"A gente divide o paciente em AD1, AD2, AD3[...]. O paciente da AD1 é aquele paciente que tem dificuldade em ir até o posto de saúde, ou ambulatório. Mas ele precisa de uma assistência médica, ou de enfermagem, ou de nutrição... pra pegar uma receita, pra poder pegar algum material, pra que ele não piore e venha a internar. Esse paciente teoricamente ... ele é acompanhado pelo... pelo SAD inicialmente, depois é transferido pra atenção primária. O paciente AD2, AD3 é aquele paciente que já é mais complexo" (M16G1)

É... e o paciente que realmente precisa dentro daqueles critérios AD1, AD2 e AD3. Então o paciente primeiro ele tem que ser elegível dentro desses critérios, ter um cuidador, ter aonde que ele vá, não precisa ser a casa dele, pode ser a casa de um familiar e.... e que a equipe consiga executar os cuidados dentro dessa casa" (M16G1)

Os gestores em saúde e os coordenadores em atenção domiciliar reforçaram os critérios clínicos como pontos para a elegibilidade do paciente no SAD, conforme os relatos a seguir:

“[...]Ter dispositivo de manutenção de vida, úlceras por pressão a partir de grau 2, usar sonda, pra adaptação de sonda [...], pra retirada de sonda também, a gente faz essa transição” (M4G1)

"O paciente que precisa de curativos que não sejam contaminados, antibioticoterapia uma vez ao dia ou de 12 em 12 horas, acompanhamento fisioterapêutico, acompanhamento nutricional, atendimento sócio assistencial, acompanhamento farmacoterapêutico", (M15G1)

"AD2 é aquele paciente que já é mais complexo. Ou seja, ele precisa de antibiótico endovenoso, por um tempo determinado, ele precisa de uma medicação anticoagulante, e... ele tem alguma escara, alguma úlcera né?! É... que precisa de acompanhamento profissional, ... paciente que tem ostomias, ou traqueostomia, ou gastrostomia, e a família ainda não sabe lidar com essas ostomias, então ele é admitido no programa. E o paciente de AD3, um paciente mais complexo, que é o de ventilação mecânica invasiva 24 horas, ... e o paciente em cuidados 
paliativos, aquele que a gente tira ele acompanha até o óbito em casa" (M16G1)

Destaca-se que os serviços e programas que ofertam a atenção domiciliar adotam diferentes critérios, majoritariamente pela condição clínica ou por idade ou ciclo vital em que os pacientes se encontram. Esse aspecto determina a posição que a atenção domiciliar irá ocupar dentro da rede de atenção à saúde ${ }^{(14)}$.

A prioridade em $\mathrm{AD}$ se torna ainda mais relevante quando há diversas comorbidades ${ }^{(15)}$. Possuir mais de uma patologia ou problema de saúde condiz com aumento dos gastos financeiros, do tempo dedicado ao cuidado e a própria produção do cuidado. Do mesmo modo, a incapacidade ou a dificuldade em realizar desde tarefas simples, como tomar banho, até atividades mais complexas, como administrar sua própria medicação e seus próprios recursos financeiros sozinho, tendem a resultar na necessidade de um cuidador, sendo esta problemática uma das grandes responsáveis pelos pedidos de inclusão em Serviços de Atenção Domiciliar ${ }^{(16)}$.

Nesse sentido, mesmo compreendendo a importância da atenção domiciliar como uma forma de acesso àqueles pacientes mais dependentes de cuidado, atualmente, os trabalhadores não têm condições de estender esse trabalho a todos ${ }^{(17)}$. Assim, salientam a importância de critérios estabelecidos para inclusão de novos pacientes no programa como forma de atender o princípio da equidade, oferecendo maior atenção aos mais necessitados.

Quanto à elegibilidade administrativa do paciente na $\mathrm{AD}$, destaca-se que a presença do cuidador representa o ponto mais complexo e importante. O cuidador é responsável por realizar todas as ações básicas para a manutenção da vida do paciente, tais como curativo, mudança de decúbito, administração e preparo de dieta e auxílio nas atividades de vida diária ${ }^{(18,19)}$. Ressalta-se a importância da presença do cuidador, que perpassa desde a avaliação da equipe quanto à elegibilidade administrativa do paciente na atenção domiciliar e na sua permanência a partir das atividades determinadas.

"Encaixou naqueles critérios e tem um cuidador, vem pra casa[...]. Mas o difícil mesmo é o cuidador” (M3G1)

"Primeira coisa: cuidador identificado. Paciente precisa ter alguém que nos ajude a cuidá-lo. A equipe não está 24 horas dentro da casa do paciente. Então, o banho, alimentação, dormir com o paciente é responsabilidade de alguém. Esse cuidador pode ser um cuidador ... um familiar que a gente vai treinar, a pessoa não precisa saber nada, a gente vai fazer isso com ela dentro do hospital. Um cuidador que é pago pela família" (M16G1)

Os resultados indicam a centralidade do cuidado na definição dos critérios de inclusão na atenção domiciliar. A equipe do SAD, desta forma, tem papel importante na relação com o cuidador, auxiliando-o, capacitando-o em procedimentos assistenciais, esclarecendo dúvidas e fornecendo suporte psicológico ${ }^{(20)}$. É primordial que os profissionais de AD estabeleçam relação de confiança com a família, como cumprir as combinações, sensibilizar-se com a situação do paciente e do cuidador e, sempre que necessário, rever o plano de cuidados ${ }^{(21)}$.

Os critérios de inclusão na $\mathrm{AD}$ estão relacionados aos aspectos administrativos, possuir domicílio com infraestrutura física compatível com a realização da $\mathrm{AD}$ (água, energia elétrica, fontes de comunicação, acesso para veículos, janelas e dimensão mínima para um leito e equipamentos). Todas as possibilidades devem ser consideradas em relação às necessidades e singularidade de cada caso atendido ${ }^{(8)}$, indicando a flexibilidade para a inclusão que deve ser discutida e avaliada pela equipe, mesmo tendo como base os protocolos e documentos que disciplinam os critérios de elegibilidade para a AD. Desta forma, cada município consegue seguir o determinado pelas normas, mas possui potência para adequar à sua realidade.

Ainda assim, está presente nos relatos dos coordenadores o reforço do aspecto normativo com o seguimento dos aspectos estabelecidos nas resolutivas do Programa no âmbito nacional. No entanto, algumas situações especiais devem ser avaliadas minuciosamente de forma conjunta entre a equipe, com abertura para exceções:

"Nós levamos em consideração as premissas do programa, que o programa determina. Mas toda regra, [...] tem sua exceção, foi o que eu te falei" (M18 P1)

Os resultados indicam que as equipes atuam com adaptações de acordo com a realidade e necessidade do paciente e consideram ainda as deficiências da rede para determinar a inclusão na AD.

"Tem sua exceção[...]. Tem pacientes que a gente sabe que é da atenção básica que poderia ser da atenção básica. Mas por algum detalhe, a gente acaba puxando" (M18P1)

Torna-se fundamental considerar todas as possibilidades com relação às necessidades da população atendida, contemplando a situação de cada paciente de forma individualizada e inclusiva, levando em consideração suas singularidades, além da capacidade e das condições do SAD em atendê-las de forma integral ${ }^{(10)}$.

Essa estratégia de se organizar para atender alguns pacientes que não se adequem ao exato perfil proposto tem como intuito o uso de ferramentas transprofissionais que possibilitam acesso assistencial com equidade ao valorizar a complexidade do paciente como ser biopsicossocial, faz com que a otimização do cuidado seja alcançada(22). Para 
que o cuidado seja efetivo, é preciso que a equipe de $\mathrm{AD}$ tenha experiência ampliada de clínica e a prática voltada para o ser humano e sua subjetividade.

No entanto, os gestores e coordenadores deixam claro que o critério de inclusão é fundamental por contribuir na estruturação do perfil dos pacientes a serem atendidos na política pública. Isto evita conflitos entre outros serviços e delimita qual serviço é mais indicado para cada paciente avaliado.

Anterior à admissão dos usuários aos serviços de atenção domiciliar, há uma avaliação de cada caso, geralmente feita em conjunto por enfermeiro e assistente social. A avaliação envolve compreender o caso em si, a estrutura do domicílio e a dinâmica familiar, possibilita também verificar a disposição e as possibilidades de execução do cuidado em casa ${ }^{(23)}$.

Segundo as orientações do programa de atenção domiciliar, existem quatro formas de captar usuários: 1) busca ativa com captação, no âmbito hospitalar; 2) busca no sistema eletrônico de pacientes com alta permanência nas unidades de internação ou de grande procura pelos serviços de urgência; 3) indicação da equipe de assistência nas unidades de internação ou pelas equipes de atenção básica; e 4) demanda espontânea em situações agudizadas, por meio de contatos telefônicos com os serviços de saúde ${ }^{(14)}$.

Os achados do estudo confirmam que as equipes adotam essas estratégias:

"É no domicilio, a gente vai no domicilio do paciente e faz essa primeira avaliação, pra saber se ele realmente se enquadra dentro do programa ou não, enquadrando a gente admite ele dali os outros profissionais passam a atender ele, não enquadrando a gente faz a contra referência pra quem enviou ele pra gente" (M9P1)

"Então antes que esse paciente receba a alta da policlínica, a nossa assistente social, a nossa fisioterapeuta, a nossa enfermeira, ela vai à residência faz uma avaliação estrutural do domicílio" (M11G1)

"Então a gente faz corrida de leito dentro do hospital do município, semanalmente, toda terça, quarta e quinta a nossa equipe está lá dentro discutindo os casos, tentando captar os pacientes. A gente tem tentado fazer essas corridas de leito dentro do hospital de clínica, ainda não tem uma equipe de desospitalização, mas a gente sempre quando identifica a gente anda dentro do hospital pra poder tentar desospitalizar e a equipe do melhor em casa do municipio também tenta sempre captar os pacientes dentro das unidades de atendimento integrado né?! que é as UAIs, e... que é referência de internação do município" (M16G1)

É importante destacar que os critérios de inclusão e de elegibilidade por vezes se confundem, e nos relatos dos gestores e coordenadores, eles se misturam quando da avaliação do paciente para o começo do atendimento no programa. Alguns critérios de inclusão também deixam margem para interpretações distintas, não sendo totalmente claros e definidos quanto à real característica que o paciente deve apresentar:

\begin{abstract}
"Os critérios de inclusão, quando a gente fala em inclusão acaba tendo uma semelhança com o critério de elegibilidade. Então o paciente, além de tudo que eu já falei sobre elegibilidade, dele estar estável, dele residir aqui, dele se, tem capacidade do autocuidado ou ter um cuidador. Existem situações que fica até fácil eu mencionar o que exclui pra gente ter a ideia, porque os critérios de exclusão são bem menores em número. Então a gente já tem uma ideia de que todo resto a gente vai poder aceitar. [...] O programa funciona 12 horas por dia, das 07 as 19 horas. Então a gente não consegue lidar com uma antibioticoterapia que seja, que é uma posologia de 6 em 6 horas, de 8 em 8 horas" (M15G1)
\end{abstract}

\section{Critérios de Alta e Exclusão}

De acordo com as normativas do Programa Melhor em Casa, a alta da atenção domiciliar refere-se ao encerramento da prestação dos serviços decorrente da melhora das condições clínicas e/ou estabilidade clínica; do agravo do quadro que justifique internação hospitalar; da mudança da área de abrangência; da solicitação de desligamento a pedido do paciente e/ou familiar ou óbito. Já os pacientes excluídos do SAD, são aqueles que descumprem os acordos assistenciais entre equipe e usuários ou cuidadores. Tendo a garantia de continuidade do atendimento deste usuário em outro serviço da rede de atenção ${ }^{(10)}$.

Verifica-se nos relatos dos gestores e coordenadores o seguimento dos critérios de alta determinado pelos documentos normativos da política de AD.

"Alta estabilidade, é... paciente que não precisa mais nem da ventilação mecânica e nem da oxigenioterapia, paciente que muda né? vai pra outro município e a gente não consegue mais acompanhar. São esses casos, paciente que melhora mesmo" (M16G1)

"Então confirmou que tá livre do microorganismo a infecção não acontece mais, então esse paciente tem tudo pra ter uma alta clínica" (M15G1)

"Alta administrativa quando você depois da terceira visita não encontra o paciente em casa, mudança de endereço" (M7G2)

"Critério de alta é essa estabilidade clínica mesmo que a gente ver que a atenção básica vai dar conta porque quando não vai dar conta, paciente é idoso, o cuidador é idoso e a família não vai dar conta, normalmente eu não recomendo dar alta" (M4G1) 
Os entrevistados relatam ainda que os pacientes ao receberem alta do programa devem ser contrarreferenciados para outro ponto da rede. A responsabilidade quanto à sequência do tratamento do paciente é apontada pelos participantes:

\begin{abstract}
"Estabilizou, a familia já está orientada, o paciente está estável, a gente contra referência para a atenção primária, ai a atenção primária recebe esse paciente, se ele agudizar, eles contra referenciam novamente" (M19G1)
\end{abstract}

É preciso modelar a rede de forma resolutiva para as demandas da população, considerando os pontos de atenção e os sistemas de apoio e logística ${ }^{(24)}$. Em casos de estabilidade ou melhora do quadro, o paciente deve ser encaminhado para a atenção básica. Já os que tiveram agravo do quadro devem ser direcionados para instituição hospitalar ou para unidade de pronto atendimento ${ }^{(8)}$.

Os resultados indicam preocupações dos participantes quanto à incerteza de continuidade do cuidado em outros pontos da rede, o que "obriga" as equipes a adiar a alta do paciente.

"a atenção básica vai dar conta porque quando não vai dar conta, paciente é idoso, o cuidador é idoso e a família não vai dar conta, normalmente eu não recomendo dar alta, por mais que todo mundo fique "ai, você tá protegendo paciente", às vezes sim porque eu acho que não tem coisa pior pra você vê que deu alta pra pessoa que queria viver e ela morreu por causa de irresponsabilidade, sei lá se é irresponsabilidade ou por negligência, né? Então quando eu vejo que não tem jeito, nós vamos pra discussão e tudo o mais e eu falo "não tem jeito, vai ter que ficar", mesmo que seja uma visita por mês" (M4G1)

"[...] a gente começa a tirar o time de campo ou a tentar dar alta [...], vamos só de 15 em 15 dias, vamos ver se ele cria autonomia de telefonar caso aconteça alguma coisa e ai a gente vê que alguns não ligam; que a gente tem que voltar uma vez por semana, porque a gente chega lá, o cuidador fala que ele foi no hospital porque sentiu isso, mas porque não ligou pra gente, sabe que tá com a gente, ai a gente começa a ter que voltar, retroceder" (M1P1)

Entre os pontos elencados nas entrevistas na exclusão, o cuidador foi o motivo mais evidenciado, seja quando o cuidado ocorre de maneira inadequada, ou quando os membros da família não estão disponíveis ou estão despreparados para essas responsabilidades. Por isso, fazse necessária a avaliação contínua da situação do paciente em $\mathrm{AD}$ e caso surja algo que comprometa o cuidado do paciente, este deverá ser discutido entre a equipe do $\mathrm{SAD}$ e familiares, podendo gerar alta e ou encaminhamento para outro ponto da rede. Quando essa situação é detectada antes da inclusão do paciente no SAD, este passa a ser um critério administrativo de exclusão.

"E pra exclusão além desses, a gente tem o paciente que não reside aqui, que não tem cuidador, que mora sozinho mas não tem a capacidade do autocuidado. [...] são critérios de exclusão, infelizmente o paciente não pode ser aderido" (M15G1)

“... o grande problema hoje não é domicilio é a questão social por que a gente vê casas precárias demais, fazendo um cuidado perfeito sabe, por que água e sabão a maioria tem o resto, a gente dá um jeito... né até energia hoje a gente tem a portaria ministerial que fala da diminuição da conta de energia para pacientes que usam equipamentos que demandam, então assim todas as adequações a gente consegue fazer, agora você obrigar uma pessoa a acolher, esse é o grande problema" (M3G1)

A presença do cuidador se faz importante, seja esse cuidador um familiar, um vizinho, um amigo voluntário ou um cuidador contratado. Uma parte do cuidado que, institucionalmente, seria realizada pela equipe de saúde passa a ser realizada pelo cuidador ou pela família. Assim é possível compreender a condição "não ter o cuidador", como critério de exclusão(23). Estes cuidadores podem sofrer com desgastes no vínculo com interferências na prestação do cuidado. Além disso, os cuidadores possuem outras atividades para além do cuidado, o que pode gerar sobrecarga e afetar o relacionamento com o paciente e o restante da família ${ }^{(25,26)}$.

A AD procura envolver, como objeto de análise e aperfeiçoamento, o trabalho de toda a equipe de assistência, e, também, dos familiares que se responsabilizam pelos cuidados ao paciente. Deve ser executada considerando os princípios éticos e legais das profissões, resguardando a intimidade e privacidade do paciente a fim de protegêlo de ações inapropriadas por parte do cuidador, sugerindo alternativas eticamente aceitáveis. A AD depende da participação de todos em um processo cooperativo, no qual a meta deve ser o cuidado ao paciente em suas demandas e necessidades, independente da classificação do cuidado $^{(27)}$.

Pode-se relacionar como limites deste estudo que os resultados descritos carregam restrições quanto ao contexto no qual o estudo foi desenvolvido. Os resultados apresentados suscitam novas questões que poderão ser respondidas com novas pesquisas neste ou em outros cenários, que permitam a comparação no contexto nacional e internacional.

\section{CONSIDERAÇÕES FINAIS}

A atenção domiciliar deve ser realizada quando as condições clínicas e administrativas do usuário permitam, 
considerando os critérios de elegibilidade, inclusão, exclusão e alta.

A presença do cuidador evidencia-se como um dos maiores determinantes para elegibilidade e exclusão do paciente, pois o mesmo representa o ator fundamental para continuidade do cuidado no domicílio, além de atuar como elo entre o paciente e a equipe.

Apesar dos critérios do SAD estarem definidos pelo Ministério da Saúde, observou-se que os gestores e coordenadores abrem exceções na inclusão e alta, considerando, a fragilidade da rede para alta e encaminhamento para outro ponto de atenção e a realidade clínica ou administrativa do paciente.

Diante dos achados, fica evidente, que somente por meio de uma rede de saúde bem estruturada e resolutiva, será possível o fortalecimento da Atenção Domiciliar como uma importante estratégia para consolidação do SUS.

\section{REFERÊNCIAS}

1. Closs VE, Schwanke CHA. A evolução do índice de envelhecimento no Brasil, nas suas regiões e unidades federativas no período de 1970 a 2010. Rev Bras Geriatr Gerontol. 2012;15(3):443-58.

2. Duarte EC, Barreto SM. Transição demográfica e epidemiológica: a epidemiologia e serviços de saúde revisita e atualiza o tema. Epidemiol Serv Saude. 2012;21(4):529-32.

3. Mendes ACG, Sá DA, Miranda GM, Lyra TM, Tavares RA. The public healthcare system in the context of Brazil's demographic transition: current and future demands. Cad Saúde Pública. 2012;28(5):955-64.

4. Silva KL, Sena RR, Braga PP, Silva PM, Souza CG. Supplementary home health care services and the inclusion of nursing in Belo Horizonte/Minas Gerais (Brazil). Acta Paul Enferm. 2012;25(3):408-14.

5. Bajotto AP, Witter A, Mahmud SJ, Sirena S, Goldim JR. Perfil do paciente idoso atendido por um programa de atenção domiciliar do sistema único de saúde em Porto Alegre, RS. Clin Biomed Res. 2012;32(3):311-7.

6. Lima AA, Spagnuolo RS, Patricio KP. Revendo estudos sobre a assistência domiciliar ao idoso. Psicol Estud. 2013;18(2):343-51.

7. Brasil. Resolução RDC n ${ }^{\circ}$ 11, de 26 de janeiro de 2006. Dispõe sobre o Regulamento Técnico de Funcionamento de Serviços que prestam Atenção Domiciliar. Ministério da Saúde: Agência Nacional de Vigilância Sanitária. Brasília. 2006. [acesso em 2016 Fev 05]. Disponível em: http://bvsms.saude.gov.br/ bvs/saudelegis/anvisa/2006/res0011_26_01_2006. html

8. Ministério da Saúde (BR), Secretaria de Atenção à Saúde, Departamento de Atenção Básica. Caderno de atenção domiciliar. Brasília: Ministério da Saúde; 2012. v.1.

9. Brasil. Portaria no ${ }^{\circ}$ 963, de 27 de maio de 2013. Redefine a Atenção Domiciliar no âmbito do Sistema Único de Saúde (SUS). Ministério da Saúde: Gabinete do Ministro. Brasília. 2013 [acesso em 2016 Mar 02]. Disponível em: http://bvsms.saude.gov.br/bvs/ saudelegis/gm/2013/prt0963_27_05_2013.html

10. Ministério da Saúde (BR), Secretaria de Atenção à Saúde, Departamento de Atenção Básica. Caderno de atenção domiciliar. Brasília: Ministério da Saúde; 2013. v.2.

11. Silva ROL. A visita domiciliar como ação para promoção da saúde da família: um estudo crítico sobre as ações do enfermeiro [tese]. Rio de Janeiro: Universidade Federal do Rio de Janeiro; 2009.

12. Lacerda MR, Giacomozzi CM, Oliniski SR, Truppel TC. Atenção à saúde no domicílio: modalidades que fundamentam sua prática. Saúde Soc. 2006;15(2):8895.

13. Bardin L. Análise de conteúdo. $7^{\mathrm{a}}$ ed. Lisboa: Edições $70 ; 2011$.

14. Ministério da Saúde (BR). Atenção domiciliar no SUS: resultados do laboratório de inovação em atenção domiciliar. Brasília: Ministério da Saúde; 2014.

15. Menezes LP. Atenção domiciliar: validação de critérios de inclusão na atenção básica de saúde [dissertação]. Porto Alegre: Universidade Federal do Rio Grande do Sul; 2014.

16. Martin JIG, Oliveira LMA, Duarte NSC. Análise da intensidade dos serviços de cuidado prestados aos Utentes Idosos do Serviço de Apoio Domiciliário. Rev Bras Geriatr Gerontol. 2010; 13(2):245-53.

17. Kerber NPC, Kirchhof ALC, Cezar-Vaz MR. Considerações sobre a atenção domiciliária e suas aproximações com o mundo do trabalho na saúde. Cad Saúde Pública. 2008;24(3):485-93.

18. Gomes WD, Resck ZMR. A percepção dos cuidadores domiciliares no cuidado a clientes com sequelas neurológicas. Rev Enferm UERJ. 2009;17(4):496-501.

19. Baptista BO, Beuter M, Girardon-Perlini NMO, Brondani CM, Budó MLD, Santos NO. Overload of family caregiver at home: an integrative literature review. Rev Gaúch Enferm. 2012;33(1):147-56. 
20. Freitas IBA, Meneghel SN, Selli L. The construction of care by the health team and the caretaker within a home-care program for bedridden patients in Porto Alegre (RS, Brazil). Ciênci Saúde Coletiva. 2010;16(1): 301-10.

21. Santos LR, Leon CGRMP, Funghetto SS. Princípios éticos como norteadores no cuidado domiciliar. Ciênc Saúde Coletiva. 2011;16(Supl 1):855-63.

22. Gallassi CV, Ramos DFH, JY Kinjo, Souto BGA. Atenção domiciliar na atenção primária à saúde: uma síntese operacional. ABCS Health Sci. 2014;39(3): 177-85.

23. Feuerwerker LM, Merhy EE. Home care's contribution to alternative health care networks: deinstitutionalization and transformation of practices. Rev Panam Salud Pública. 2008;24(3):180-9.

24. Mendes EV. O cuidado das condições crônicas na atenção primária à saúde: o imperativo da consolidação da estratégia da saúde da família. Brasília: Organização Pan-Americana da Saúde; 2012.
25. Venancio-Tamanini RA. Representações sociais de cuidadores domiciliares de idosos pós acidente vascular encefálico [dissertação]. Ribeirão Preto: Universidade de São Paulo; 2013.

26. Vieira L, Nobre JRS, Bastos CCBC, Tavares KO. Cuidar de um familiar idoso dependente no domicílio: reflexões para os profissionais da saúde. Rev Bras Geriatr Gerontol. 2012;15(2):255-64.

27. Paiva PA, Costa FM, Vieira MA, Silva SSS, Dias OV. Internações sensíveis à atenção domiciliar em um hospital de ensino em Montes Claros - MG, Brasil. Rev Unimontes Científica. 2015;17(2):14-26.

\section{Endereço para correspondência:}

Patrícia Alves Paiva

Universidade Estadual de Montes Claros - UNIMONTES

Rua Tiago Andrade Amaral, 203

Bairro: Vila Atlântida

CEP: 39401-145 - Montes Claros (MG) - Brasil

E-mail: patrícia.alves.paiva@hotmail.com 